MYCN gene which was confirmed by FISH. Tumor MYCN amplification status is unknown.

Conclusion Management of perinatal neuroblastoma includes close observation with therapeutic intervention reserved for advanced stage and/or clinical progression. Two of the five cases of neuroblastomas described in patients with dysmorphic features and a germline partial $2 p$ duplication, were detected perinatally. All patients had an aggressive clinical course. In the subset of patients with perinatal neuroblastoma and multiple congenital anomalies, FISH or aCGH testing for partial $2 p$ gain may identify those who may need more aggressive management.

\section{IGF1R DELETION IN A PRENATAL CASE}

doi:10.1136/archdischild-2012-302724.0556

'M Kammoun, 'R Hichri, 'H Hannechi, ${ }^{2} S$ Mechmech, 'D Hmida, ${ }^{2} \mathrm{M}$ Bibi, 'A Saad, 'S Mougou. 'Departments of Cytogenetics and Reproductive Biology; '2Departments of Gynecology Obstetrics, Farhat Hached University Teaching Hospital, Sousse, Tunisia

Small fetuses constitute a large group including healthy small fetuses, fetuses suffering from utero-placental insufficiency or chromosomal abnormality. In the latter case, chromosomal rearrangements may be showed by fetal karyotyping. However, they are usually cryptic and require to be detected by molecular investigations.

In this study, we report on a case of 15q26 microdeletion diagnosed prenatally in a fetus found to have severe intra uterine growth retardation, congenital diaphragmatic hernia and polycystic kidneys identified at 28 weeks of gestation by ultrasonography.

Amniocentesis was performed and revealed a normal karyotype of 46, XX. However, phenotypic features let us to test the $15 \mathrm{q} 26$ microdeletion which was confirmed by IGF1R FISH probe.

$I G F 1 R$ gene is involved in pre and post natal growth. Monozygosity for IGF1R gene is responsible for growth delay, microcephaly, mental retardation, micrognathia and deafness. Congenital diaphragmatic hernia and polycystic kidneys are common findings in 15 q26 microdeletion. Genotype phenotype correlation localized critical region implicated in diaphragmatic hernia in 15q26.1-q26.2. NR2F2, CHD2, RGMA and SIAT8B are considered to be most likely candidate genes. Genes involved in kidney defect are little studied. In our case, the deletion must be extended to $15 \mathrm{q} 26.1$. Molecular characterization by CGH array is considered to refine genotype phenotype correlation and to localize candidate genes for diaphragmatic hernia and kidney defect.

$15 q 26$ microdeletion causes intra uterine and post natal growth retardation variably associated to other malformations. We highlight the importance of molecular analysis in the prenatal diagnosis of cryptic chromosomal abnormalities.

\section{SUPPOSED"SATELLITE 80" CHROMOSOME ASSOCIATED TO A GROWTH RETARDATION AND NEUROLOGY DEVELOPMENT DELAY}

doi:10.1136/archdischild-2012-302724.0557

'MT Garcia, 'L Rodriguez, 'M Benedit, 2 Llana, 'L Golmayo. 'Hospital Madrid Sanchinarro; ${ }^{2}$ Hospital Madrid Torrelodones, Madrid, Spain

Polymorphisms are cytogenetic laboratory findings that in most cases are inherited without clinical repercussion, when they are found in a karyotipe usually none additional studies are carried out. One of the most common is the presence of a satellite (NOR: nucleolus organizer regions) on a non-acrocentic chromosome. These extra NOR regions generally result from a translocation between a NOR region of an acrocentric chromosome and a non-acrocentric chromosome and are easily confirmed by NOR-bands in laboratory. These translocations are usually terminal and have been described on multiple chromosomes, the most frequent involves de $\mathrm{Y}$ chromosome. We present a case with a satellite chromosome and clinical expression. A 24 months old girl was referred to our hospital with deeply postnatal growth retardation, dymosphic features, motor delay and seizures. Cytogenetic studies were requested and a satellite in a chromosome 8 (8qs) was founded. FISH showed on the abnormal chromosome 8 a hybridization signal much bigger than the one detected on the normal homologue, these suggest a 8q24 inverted duplication. Array-CGH analysis at $40 \mathrm{~Kb}$ resolution confirmed the duplicated region of $17.2 \mathrm{Mb}$ in 8q24.1-q24.3, associated to a terminal $55 \mathrm{~Kb}$ deletion. Furthermore, a triplicated region of $76 \mathrm{~Kb}$ between the duplicated and deleted regions was detected. The diagnosis is a $46, \mathrm{XX}$ invdupdel (8) de novo. With this case we want to show that properly cytogenetic studies are very important in order to define the "supposed satellite chromosome" and to find other chromosome abnormalities that could explain the clinical findings of some patients.

\section{PRADER-WILLI SYNDROME DUE TO MATERNAL UNIPARENTAL DISOMY FOLLOWING ASSISTED REPRODUCTIVE TECHNOLOGY}

doi:10.1136/archdischild-2012-302724.0558

0 Cogulu, T Atik, E Karaca, H Onay, F Ozkinay. Ege University Faculty of Medicine, Izmir, Turkey

Assisted Reproductive Technology (ART) refers to methods used to provide pregnancy by the manuplation of sperm and ova in vitro. Concern has been raised recently on the safety about the health of children born after ART. Although abnormal genomic imprinting has been shown to be involved in a number of genetic syndromes identified in the pregnancies with ART, there is a lack of evidence linking ART with the Prader-Willi syndrome (PWS) which is caused by the lack of expression of paternally inherited genes on chromosome 15q11-q13. Paternal microdeletion (70\%), maternal uniparental disomy (25-30\%), and imprinting defect $(2-5 \%)$ are the main causes of PWS. Here 2-year-old male/female twins, male with upd(15)mat, born to a nonconsanguineous parents following ART is presented. The proband was between 90-97 percentile for height, weight and head circumference on admission. Physical examination showed hypotonia, almondshaped palpebral fissures, low-set ears, long philtrum, small hands and feet. His history revealed feeding difficulties and delayed developmental milestones. MRI, EEG and EMG were normal. Echocardiography showed supravalvular pulmonary stenosis, which disappeared with aging. Pelvicaliectasis was demonstrated sonographically. Serum biochemical tests, thyroid function tests and amino acid chromatography were normal. Karyotype analysis, FISH analysis for PWS and subtelomeric regions were normal. DNA methylation analysis revealed maternal uniparental disomy. In conclusion this case contributes to the literature in two ways, first the importance of clinical evaluation with molecular testing in the diagnosis of PWS is pointed out, and second ART may have an effect on the occurrence of imprinted diseases such as PWS.

\section{WILLIAMS SYNDROME PRESENTING WITH FINDINGS CONSISTENT WITH ALAGILLE SYNDROME}

doi:10.1136/archdischild-2012-302724.0559

IP Sakhuja, 'H Whyte, ${ }^{2,3} \mathrm{D}$ Chitayat. ${ }^{1}$ Acute Care Transport Services, Division of Neonatology; ${ }^{2}$ Division of Clinical and Metabolic Genetics, The Hospital for Sick 